\title{
SULLA RISULTANTE DI DUE EQUAZIONI DI 4: GRADO
}

$\mathrm{N} O \mathrm{~T}$ A

DEL CAY. FR. FAA' DI BRUNO.

Essendo occorsi varii errori nell'espressione della risultante suddetta da me data negli Annali (Ottobre 1855), credo ntile di qui rettificarli a comodo dei leltori, ricorrendo ad una scritturazione ideata dal Cuyley. Si ha dunque per questa risultante l'espressione

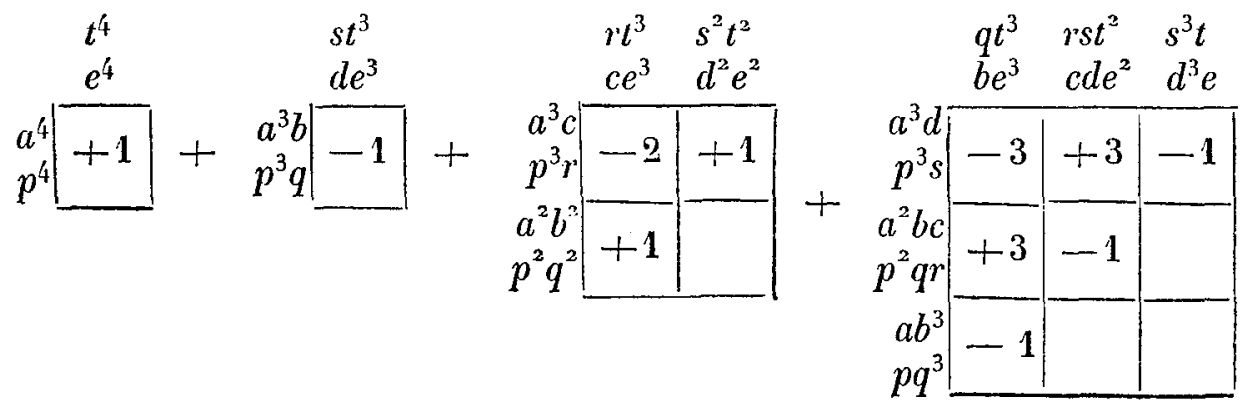

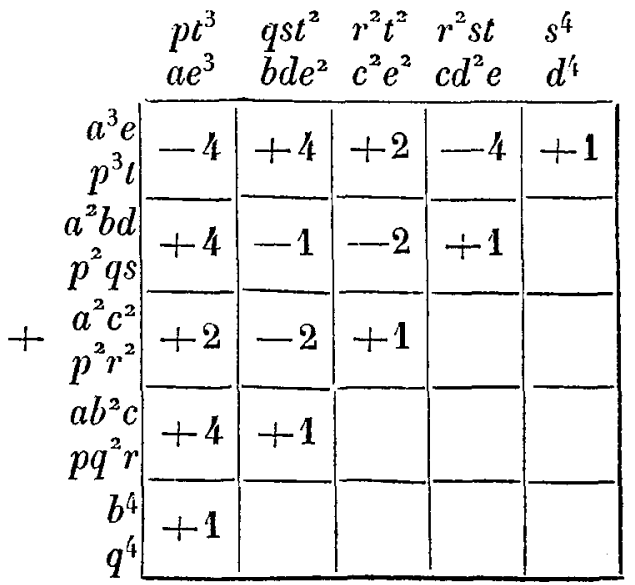

$$
\begin{aligned}
& p s t^{2} \quad q r t^{2} \quad q s^{2} t \quad r^{2} s t \quad r s^{3} \\
& \begin{array}{lllll}
a d e^{2} & b c e^{2} & b d^{2} e & c^{2} d e & c d^{3}
\end{array} \\
& \begin{array}{rr|r|r|r|r|r|}
a^{2} b e & -1 & -5 & +1 & +3 & -1 \\
p^{2} q t & & & & & \\
a^{2} c d & -5 & +1 & +2 & -1 & \\
p^{2} r s & & & & & \\
+\quad a b^{2} d & +1 & +2 & -1 & & \\
p q^{2} s & & & & & \\
a b c^{2} & +3 & -1 & & & \\
p q r^{2} & & & & & \\
b^{3} c & -1 & & & & \\
q^{3} r & -1 & & & & \\
\hline
\end{array}
\end{aligned}
$$


PURA ED APPLICATA.

$p r t^{3} \quad q^{2} t^{2} \quad p s^{2} t \quad q r s t \quad r^{3} t \quad q s^{3} \quad r^{2} s^{2}$

$p q t^{2} \quad p r s t q^{2} s t \quad q r^{2} t \quad p s^{3} \quad q r s^{2} r^{3} s$ $\begin{array}{lllllll}a c e^{2} & b^{2} e^{2} & a d^{2} e & b c d e & a^{3} e & b d^{3} & c^{2} d^{2}\end{array}$

\begin{tabular}{|c|c|c|c|c|c|c|c|}
\hline & $\begin{array}{l}p q t^{2} \\
a b e^{2}\end{array}$ & $\begin{array}{l}\text { prst } \\
\text { acde }\end{array}$ & $\begin{array}{l}q^{2} s t \\
b^{2} d e\end{array}$ & $\begin{array}{l}q r^{2} t \\
b e^{2}\end{array}$ & $\begin{array}{l}p s^{3} \\
a d^{3}\end{array}$ & $\begin{array}{l}q r s^{2} \\
b c d d^{2}\end{array}$ & $\begin{array}{l}r^{3} s \\
c^{3} d\end{array}$ \\
\hline $\begin{array}{c}a^{2} d e \\
p^{2} s t\end{array}$ & +5 & +2 & -5 & +1 & -3 & +3 & -1 \\
\hline $\begin{array}{l}a b c e \\
p q r t\end{array}$ & +2 & -8 & +1 & +2 & +3 & -1 & \\
\hline $\begin{array}{l}a b d^{2} \\
p q s^{2}\end{array}$ & -5 & +1 & +2 & -1 & 0 & & \\
\hline $\begin{array}{l}a c^{2} d \\
p r^{2} s\end{array} \mid$ & +1 & +2 & -1 & -1 & & & \\
\hline $\begin{array}{c}b^{3} e \\
q^{3} t\end{array}$ & -3 & +3 & 0 & & -1 & & \\
\hline $\begin{array}{c}b^{2} c d \\
q^{2} r s\end{array}$ & +3 & -1 & & & & & \\
\hline $\begin{array}{c}b c^{3} \\
g r^{3}\end{array}$ & -1 & & & & & & \\
\hline
\end{tabular}

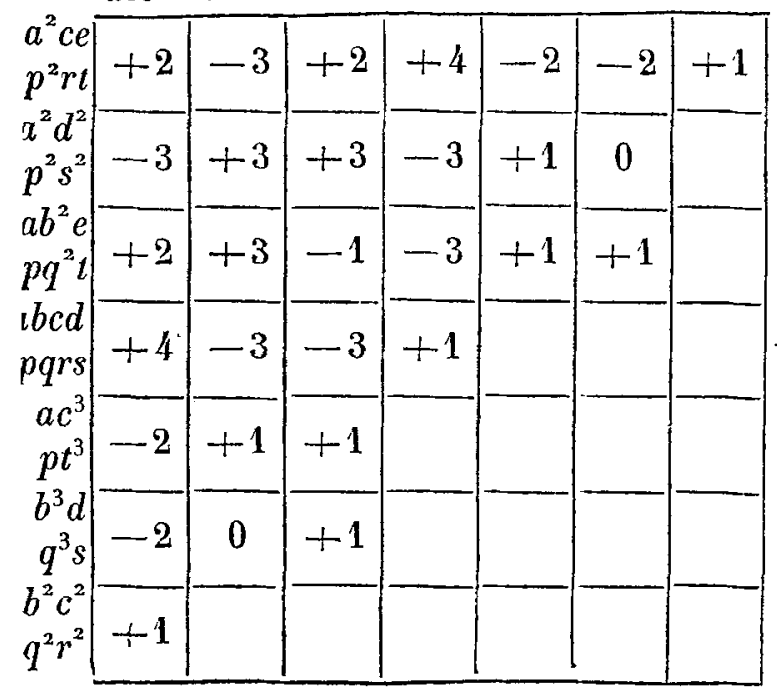

\begin{tabular}{|c|c|c|c|c|c|c|c|c|}
\hline & $p^{2} t^{2}$ & pqst & $p r^{2} t$ & $q^{2} r t$ & $p^{p} s^{2}$ & $q^{2} s^{2}$ & $q r^{2} s$ & $r^{4}$ \\
\hline$a^{2} e^{2}$ & +6 & -8 & -4 & +4 & +4 & +2 & -4 & +1 \\
\hline$a b d e$ & -8 & +10 & & -1 & -1 & -1 & +1 & \\
\hline$a c^{2} e$ & -4 & & +4 & -2 & -2 & +1 & & \\
\hline$a c d^{2}$ & +4 & -1 & -2 & & +1 & & & \\
\hline$b^{2} c e$ & +4 & -1 & -2 & +1 & & & & \\
\hline$b^{2} d^{2}$ & +2 & -1 & +1 & & & & & \\
\hline$b c^{2} d$ & -4 & +1 & & & & & & \\
\hline & +1 & & & & & & & \\
\hline
\end{tabular}

avvertendo di moltiplicare solo insieme $\mathrm{i}$ termini letterali superiori coi superiori e gli inferiori cogli inferiori. Per esempio dal $7^{\circ}$ quadro si ricaverà che i prodotli $a b^{2} e q r s t$, $b c d e p q^{2} t$ hanno -3 per coefficiente numerico. Si seorge altresi che i coefficienti numerici sono simmettrici rispelto alle diagonali \, come deve esscre da quanto dicemmo in quella nota. 